\title{
T-bet expression by Th cells promotes type 1 inflammation but is dispensable for colitis
}

\author{
J Zimmermann ${ }^{1}$, AA Kühl ${ }^{2}$, M Weber ${ }^{1}$, JR Grün ${ }^{1}$, J Löffler ${ }^{1}$, C Haftmann ${ }^{1}$, R Riedel ${ }^{1}$, P Maschmeyer ${ }^{1}$,

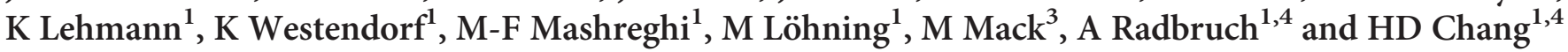

The transcription factor T-bet is highly expressed by Th cells isolated from the inflamed intestine of Crohn's disease patients, and has been regarded a critical driver of murine T cell-induced colitis. However, we show here that T-bet expression by Th cells is not required for the manifestation of T-cell-induced colitis in the presence of segmented filamentous bacteria and Helicobacter hepaticus. T-bet expression by Th cells controls their survival and localization, their repertoire of chemokine and chemokine receptor expression, the accumulation of monocytes and macrophages in the inflamed colon, and their differentiation to the M1 type, i.e., type 1 inflammation. Nevertheless, T-bet-deficient Th cells efficiently induce colitis, as reflected by weight loss, diarrhea, and colon histopathology. T-bet-deficient Th cells differentiate into Th1/17 cells, able to express IFN- $\gamma$ and IL-17A upon restimulation. While neutralization of IL-17A exacerbated colitis induced by wild-type or T-bet-deficient Th cells, neutralization of IFN- $\gamma$ completely abolished colitis.

\section{INTRODUCTION}

Inflammatory bowel diseases, comprising Crohn's disease (CD) and ulcerative colitis, affect an increasing number of people in the western world and are currently not curable. ${ }^{1}$ Although the exact etiology remains elusive, it has become clear from studies using immunoablation and autologous stem cell transplantation that the immune system is critically involved in the pathogenesis and the perpetuation of chronic inflammation. ${ }^{2}$ Antibody-mediated depletion of Th cells ameliorated the disease, pointing to a role for Th cells. ${ }^{3}$ In immunodeficient $\mathrm{Rag}^{-1-}$ mice, lacking $\mathrm{B}$ and $\mathrm{T}$ cells, inflammatory bowel disease can be induced by adoptive transfer of naïve CD4+ $\mathrm{CD}_{45 \mathrm{RB}}{ }^{\text {hi }}$ Th cells. ${ }^{4}$ In the absence of regulatory T cells, the naïve Th cells react against components of the microbiota and cause chronic inflammation, characterized by weight loss, diarrhea, and pathological changes of the colon..$^{5}$ Th cells isolated from the inflamed colon express the transcription factor T-box expressed in T cells (T-bet), ${ }^{6}$ a transcription factor that directs the differentiation program of Th1 cells. ${ }^{7}$ Because ectopic expression of T-bet is sufficient to drive the differentiation of activated naïve Th cells towards the Th1 lineage and because genetic deletion of $T b \times 21$ (i.e., gene encoding T-bet) precludes Th1 differentiation, T-bet is considered to be the "master" transcription factor of the Th1 lineage. ${ }^{7-9}$ T-bet directly activates Th1-specific genes, including those encoding cytokines like Ifng and chemokine receptors critical for homing to inflamed tissues, like $\operatorname{Cxcr} 3$ and $C c r 5 .^{10}$

Early evidence for a role for T-bet in CD pathogenesis was provided by studies revealing high expression of $\mathrm{T}$-bet and interferon (IFN) $-\gamma$ in colonic Th cells of CD patients. ${ }^{6,11}$ In the T-cell transfer colitis model, T-bet-deficient Th cells could not induce colitis ${ }^{6}$ and antibody-mediated blockade of IFN- $\gamma$ prevented colitis. $^{12}$ In contrast, however, Simpson and colleagues $^{13}$ demonstrated efficient induction of colitis by IFN- $\gamma$-deficient Th cells and Gökmen et al. ${ }^{14}$ reported weight loss and colon thickening when inducing colitis with T-bet-deficient Th cells. Moreover, several groups showed that Th cells impaired in Th17 differentiation, i.e., lacking ROR- $\gamma$ t or STAT-3 cannot induce colitis, ${ }^{15,16}$ suggesting that either both, Th1 and Th17 cells, are required to induce colitis in cooperation, or that there are alternative pathways to induce colitis, one dependent on T-bet and one not.

Here, we show that T-bet-deficient Th cells can efficiently induce colitis, on the C57BL/6 genetic background and in the

\footnotetext{
${ }^{1}$ Deutsches Rheumaforschungszentrum Berlin (DRFZ), an Institute of the Leibniz Association, Berlin, Germany. ${ }^{2}$ Charité Universitätsmedizin, Campus Benjamin Franklin, Berlin, Germany and ${ }^{3}$ Universitätsklinikum Regensburg, Regensburg, Germany. Correspondence: H-D Chang (chang@drfz.de)

${ }^{4}$ These authors contributed equally to this work. 
presence of segmented filamentous bacteria (SFB), which were shown to be pivotal for the induction of Th17 cells and the development of colitis. ${ }^{17,18}$ Although the clinical parameters of weight loss and diarrhea were similar, the overall scores of histopathology of the colon were slightly reduced in colitis induced by T-bet-deficient Th cells compared with wild-type (WT). Colitis induced by T-bet-deficient Th cells was characterized by a reduced accumulation of Th cells in the colon but enhanced coexpression of IFN- $\gamma$ and interleukin (IL)-17, i.e., enhanced Th1/17 differentiation. T-bet deficiency in Th cells resulted in reduced recruitment of monocytes/ macrophages into the colon and their differentiation to M1 cells, which was also mirrored when Th cells were unable to express IFN- $\gamma$, demonstrating that both T-bet and IFN- $\gamma$, when expressed by Th cells, are not required for colitis induction but have a non-redundant role in orchestrating type 1 inflammation. Interestingly, colitis induced by wild-type or T-bet-deficient Th cells was exacerbated when IL-17A was neutralized, but completely abolished, when IFN- $\gamma$ was neutralized, demonstrating that IFN- $\gamma$ as such, but not necessarily from $\mathrm{Th}$ cells, is required for the induction of colitis.

\section{RESULTS}

\section{T-bet-deficient Th cells induce colitis}

Upon transfer of $4 \times 10^{5} \mathrm{CD} 4+\mathrm{CD} 45 \mathrm{RB}^{\text {hi }} \mathrm{CD} 25-$ Th cells of C57BL/6 mice into syngenic $\mathrm{Rag}^{-/-}$recipients, the host mice developed colitis within 2-3 weeks. All Rag1 ${ }^{-1}$ recipients were colonized with fecal microbiota containing Helicobacter hepaticus and SFB to ensure the presence of know colitogenic bacteria. ${ }^{17,19}$ Th cells isolated from the inflamed colon (between day 16 and day 21) all expressed T-bet, as demonstrated by intracellular immunofluorescence staining (Figure 1a). These data are in line with previous reports postulating a role for T-bet in disease pathogenesis. ${ }^{6}$ To validate this role of T-bet, we compared the potential of WT and Tbx21 $1^{-/}$Th cells to induce colitis. Tbx21 $1^{-/-}$Th cells caused weight loss and diarrhea comparable with WT Th cells (Figure 1b, c). Although histopathology scores of $T b \times 21^{-/-}$Th-cell-induced colitis were significantly reduced compared with WT, they clearly differed from those of a healthy colon (Figure 1d, e). Submucosal and mucosal inflammatory infiltration, crypt abscesses, and goblet cell depletion were significantly reduced in $T b \times 21^{-1-}$ Th-cell-induced colitis, compared with WT, while epithelial hyperplasia and the size of the affected area were similar (Figure 1d and Supplementary Figure S1 online). In conclusion, CD $4+\mathrm{T}$ cells do not require T-bet to induce transfer colitis.

\section{T-bet controls numbers and position of Th cells in colitis} In established colitis (days 16-21), absolute numbers of Tbx21-1- Th cells in the colon, spleen, and mesenteric lymph nodes were significantly reduced compared with WT, while in the small intestine, they were significantly increased (Figure 2a). The total number of Th cells in all organs analyzed was significantly lower in $T b \times 21^{-/-}$Th-cell-induced colitis as compared with colitis induced by WT Th cells (Supplementary
Figure S2a). We could not detect differences in the frequencies of WT and Tbx21 ${ }^{-/-}$Th cells in cell cycle, i.e., cells expressing Ki-67 (Figure 2b). However, the ratio of Bim to $\mathrm{Bcl}-2$, which is critical for survival of the cells, ${ }^{20}$ was higher in Tbx $21^{-1-}$ Th cells as compared with WT Th cells, isolated from the inflamed colon, before and after restimulation with phorbol 12-myristate 13-acetate (PMA)/ionomycin (Figure 2c and Supplementary Figure S2b). An increase in that ratio renders Th cells prone to apoptosis. Indeed, whereas frequencies of apoptotic Th cells, staining with Annexin-V, were comparable directly ex vivo, they were significantly increased in $T b \times 21^{-/-}$vs. WT Th cells after restimulation with PMA/ionomycin (Figure 2d and Supplementary Figure S2c). Together, T-bet promoted the survival of Th cells and prevented their accumulation in the small intestine.

\section{T-bet-deficient Th cells differentiate into Th1/17 cells maintaining IFN- $\gamma$ expression}

When analyzed during established colitis (day 16-21), WT Th cells isolated from the colon uniformly expressed T-bet and $5-10 \%$ of them coexpressed ROR- $\gamma$ t. Among T-bet-deficient Th cells, 60\% expressed ROR- $\gamma \mathrm{t}$ (Figure 3a, b). There was no difference in the frequency of Foxp3- or GATA-3-expressing Th cells between WT and Tbx21 ${ }^{-/-}$Th-cell-induced colitis (Supplementary Figure S3a). After restimulation with PMA/ionomycin, an average of $66 \pm 3 \%$ of colonic Th cells from WT colitis expressed IFN- $\gamma$, and $6.6 \pm 0.5 \%$ expressed IL-17A, about half of them together with IFN- $\gamma$ (Figure 3c, d). Of the $T b \times 21^{-/-}$Th cells, $46.1 \pm 1.6 \%$ expressed IFN- $\gamma$ and $66.3 \pm 2.1 \%$ expressed IL-17A, and about half of them coexpressed IFN- $\gamma$ (Figure 3c, d). Whereas frequencies of cells expressing granulocyte-macrophage colony-stimulating factor, IL-4, IL-10, and tumor necrosis factor were comparable between WT and $T b \times 21^{-1-}$, there was a significant increase in the frequency of cells expressing IL-13, IL-17F, and IL-22 (Supplementary Figure S3b). The protein concentration of IFN- $\gamma$ in supernatants of colon explant cultures was decreased from $8.5 \pm 2.4 \mathrm{pg} \mathrm{ml}^{-1}$ in WT to $0.6 \pm 0.1 \mathrm{pg} \mathrm{ml}^{-1}$ in Tbx21-1- Th-cell-induced colitis, per $1 \mathrm{mg}$ tissue, while IL-17A was increased from $1.0 \pm 0.3 \mathrm{pg} \mathrm{ml}^{-1}$ to $45.7 \pm 10.2 \mathrm{pg} \mathrm{ml}^{-1}$ (Figure 3e).

To obtain further insights into the role of T-bet for the phenotype of colitic Th cells, we generated transcriptomes from WT and Tbx21 $-1-$ Th cells isolated from the inflamed colon and restimulated ex vivo. Above a threshold of twofold, 509 genes were upregulated in Tbx21 $1^{-1-}$ vs. WT Th cells and 210 genes were downregulated (Figure 4a). Significantly increased expression of Il22, Ill7a, Rora, Rorc, and Il23r underpinned enhanced Th17 differentiation of Tbx21-/- vs. WT Th cells (Figure 4b, c). Levels of Batf, Irf4, and Il21 were not significantly different between WT and Tbx21 ${ }^{-I-}$ Th cells (Supplementary Figure S4a,b). Gata3 expression was not significantly different ex vivo but increased 1.9-fold in Tbx21 ${ }^{-1-}$ Th cells vs. WT after restimulation with PMA/ionomycin (Supplementary Figure S4c). While mRNA levels for Il4 and Il5 were upregulated about 10 -fold in T-bet-deficient Th cells vs. WT, their absolute levels in 
a

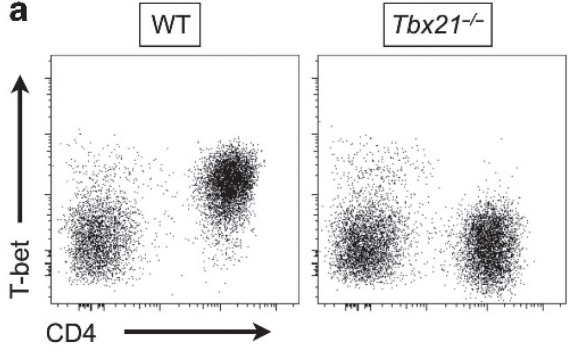

b

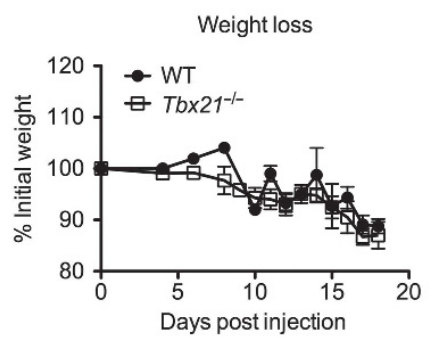

C

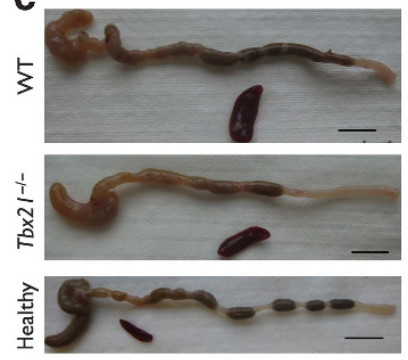

e

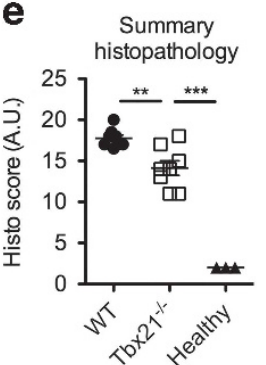

Figure 1 T-bet is dispensable for the colitogenic potential of CD4 + T cells. Naïve WT and Tbx21-1- CD4 + T cells were transferred into Rag ${ }^{-1-}$ recipients. (a) LPLs from the inflamed colon stained for CD4 and T-bet (day 18 after transfer). (b) Weight loss relative to starting weight. (c) Macroscopic pictures of colons, scale bars are $10 \mathrm{~mm}$. (d) H\&E staining of colons, scale bars are $100 \mu \mathrm{m}$. (e) Summary of the histopathology score. $n=8 \mathrm{mice}$ per group from two out of five experiments with similar results. ${ }^{\star} P<0.05,{ }^{\star \star} P<0.01$, ${ }^{\star \star \star} P<0.001$ by Student's $t$ test for independent samples. H\&E, hematoxylin and eosin; LPL, lamina propria leukocyte; WT, wild type.

Tbx21-1- Th cells were more than 20-fold lower compared with those of $I l 17 a, I l 21$, or Il22 (Supplementary Figure S4b). There was no significant difference in Eomes, Foxp3, Runx1, or Runx3 expression (Supplementary Figure S4a).

In line with the preferential positioning of $T b \times 21^{-/-}$Th cells in the small intestine (Figure 2a), these cells expressed enhanced levels of Ccr6 and Ccr9, two chemokine receptors critical for homing to the small intestine, ${ }^{21}$ as compared with WT Th cells (Figure 4d). Expression of the chemokine receptors $C x c r 3$ and Ccr5, two chemokine receptors mediating homing of Th cells to the inflamed colon, ${ }^{10}$ was lower in Tbx21 $1^{-1-}$ Th cells than in WT Th cells (Figure 4d).

Between T-bet-deficient and -sufficient Th cells, several chemokines were differentially regulated. Expression of monocyte-attracting chemokines, Ccl3, Ccl4, and Ccl5, and T cell-attracting $X c l 1$ was reduced in $T b \times 21^{-1-}$ compared with WT Th cells (Figure 4e). Expression of $\mathrm{Cxcl} 3$ and $\mathrm{Cxcl5}$, known for their neutrophil-attracting potential, was higher in Tbx21-1- Th cells than in WT Th cells (Figure 4e). Overall, T-bet deficiency caused a strong skew towards Th17 differentiation with maintenance of IFN- $\gamma$ expression and differential regulation of both cytokines and chemokines.

\section{T-bet expression by Th cells controls monocyte/ macrophage numbers and differentiation in the inflamed colon}

The composition of the colonic inflammatory infiltrate was different between colitis caused by WT and Tbx21-1- Th cells. Siglec-F + eosinophils were increased in frequency from $3.0 \pm 0.3 \%$ to $8.3 \pm 0.4 \%$ and in absolute numbers from
$3.0 \pm 0.2 \times 10^{5}$ to $4.3 \pm 0.4 \times 10^{5}$ in $T b \times 21^{-/-}$vs. WT Th-cell-induced colitis (Figure 5a-c). Ly-6G + neutrophils were significantly increased in frequency from $8.4 \pm 0.0 \%$ to $15.3 \pm 0.7 \%$ in $T b \times 21^{-1-}$ compared with WT Th-cell-induced colitis, but their absolute numbers did not differ significantly $\left(8.5 \pm 0.8 \times 10^{5}\right.$ vs. $8.3 \pm 1.0 \times 10^{5}$, Figure 5a-c). We used the marker combination CD64 and Ly-6C to identify monocytes and macrophages. Frequencies and absolute numbers of CD64 + Ly-6C $\mathrm{Ci}^{\mathrm{i}}$ monocytes were decreased in $\mathrm{Tb} \times 21^{-1-}$ vs. WT Th-cell-induced colitis from $48.4 \pm 3.6 \%$ to $21.9 \pm 1.9 \%$ and from $5.5 \pm 0.9 \times 10^{6}$ to $1.2 \pm 0.2 \times 10^{6}$, respectively (Figure $5 \mathbf{a}-\mathbf{c})$. Whereas macrophages were present in both types of colitis at frequencies of about $16 \%$, their absolute numbers were decreased from $1.4 \pm 0.1 \times 10^{6}$ in WT Th-cell-induced colitis to $0.8 \pm 0.1 \times 10^{6}$ in colitis induced by $T b \times 21^{-/-}$Th cells (Figure $5 \mathbf{a a}-\mathbf{c})$. In the spleens of colitic mice, absolute numbers of eosinophils and neutrophils were increased in $T b \times 21^{-1-}$ Th-cell-induced colitis vs. WT, while numbers of monocytes and macrophages were comparable (Supplementary Figure S5).

To test whether besides absolute numbers also the phenotype of monocytes/ macrophages is influenced by T-bet expression in Th cells, we generated transcriptomes of sorted colonic CD64 + Ly-6C ${ }^{\text {hi }}$ monocytes and CD64 + Ly-6C ${ }^{\text {low }}$ macrophages from WT and Tbx21-1- Th-cell-induced colitis. Above a threshold of twofold, 47 genes were upregulated and 103 genes were downregulated in monocytes and 128 upregulated and 144 downregulated in macrophages from Tb $\times 21^{-1-}$ Th-cell-induced colitis vs. WT Th-cell-induced colitis (Figure 6a, b). Genes characteristic for classical or M1 

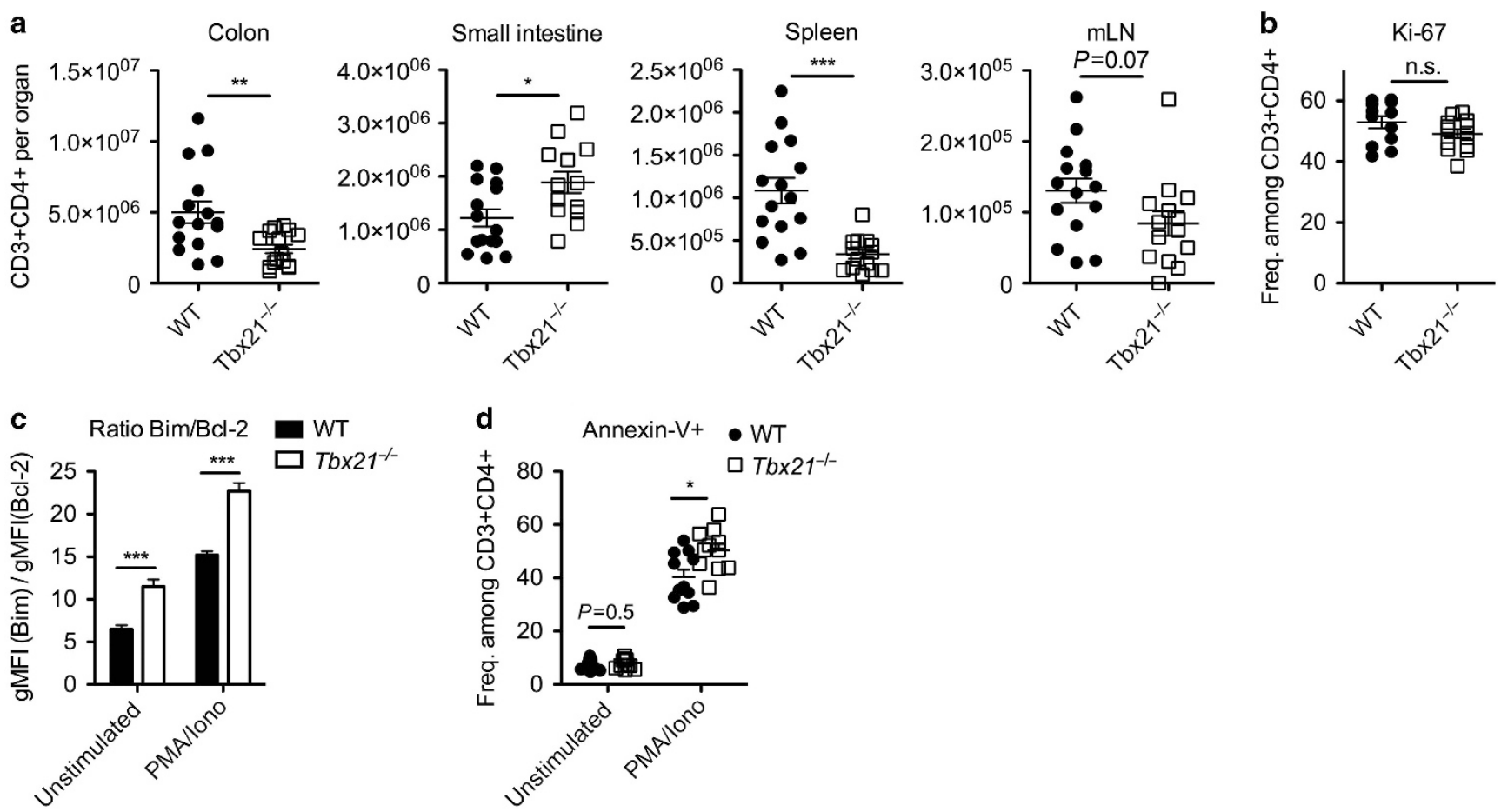

Figure 2 T-bet controls numbers and localization of Th cells. Naïve WT and Tbx21-1- CD4 + T cells were transferred into Rag ${ }^{-1-}$ recipients. (a) Absolute numbers of CD4 + Th cells in various organs of colitic mice (day 16-21 after transfer) for $n=15$ mice per group from four independent experiments. (b) Frequency of Ki-67 + cells among colonic Th cells as determined by intracellular staining for $n=12-13$ mice per group from three of more than five independent experiments with similar results. (c) Colonic Th cells were stained intracellularly for Bim and Bcl-2 directly ex vivo or after $4 \mathrm{~h}$ restimulation with PMA/ionomycin. The ratio of the gMFI of Bim and Bcl-2 are depicted for $n=16$ mice per group from two independent experiments. (d) Colonic Th cells were stained for Annexin- $V$ directly ex vivo or after $4 \mathrm{~h}$ of restimulation with $\mathrm{PMA} /$ /ionomycin. Frequencies of Annexin- $\mathrm{V}+\mathrm{cells}$ are shown for $n=11$ mice per group from two independent experiments. ${ }^{\star} P<0.05,{ }^{\star \star} P<0.01,{ }^{\star * \star} P<0.001$ by Student's $t$ test for independent samples. gMFI, geometric mean fluorescence intensities; PMA, phorbol 12-myristate 13-acetate; WT, wild type.

macrophage polarization like Fpr1, Nos2, and Il12a were significantly decreased in macrophages and monocytes from Tbx $21^{-1-}$ compared with WT Th-cell-induced colitis (Figure 6c). Conversely, genes typical for alternatively activated or M2 macrophages like Arg1, Mmp13, Irf4, Il4i1, Ccl22, and Ccl24 were significantly increased in macrophages and monocytes from $T b \times 21^{-1-}$ compared with WT Th-cell-induced colitis (Figure 6c). These data demonstrate that T-bet expression by Th cells directs the accumulation of monocytes and macrophages in the colon and their polarization to M1 cells.

To address the role of monocytes and macrophages for colitis pathology, we depleted monocytes by daily intraperitoneal (i.p.) injections of an anti-CCR2 antibody beginning on day 7 after colitis induction. On day 14, colonic monocyte and macrophage numbers were reduced by about $60 \%$ and $65 \%$, compared with isotype control, respectively (Figure $\mathbf{6 d}$, e). Numbers of monocytes and macrophages in monocyte-depleted WT Thcell-induced colitis were similar to those observed in $T b \times 21^{-1-}$ Th-cell-induced colitis without monocyte depletion (Figure 6d, e). Weight loss, diarrhea, and colon histopathology scores were not significantly different between monocyte-depleted mice and isotype controls (Figure 6f, Supplementary Figure S6). However, the overall numbers of infiltrating cells as well as $\mathrm{CD} 4+\mathrm{T}$ cells, determined by flow cytometry, were significantly reduced in monocyte-depleted colitic mice compared with isotype control-treated mice (Figure $\mathbf{6 g}$, h). Both infiltrate and numbers of $\mathrm{CD} 4 \mathrm{~T}$ cells were similar between monocytedepleted WT Th-cell-induced colitis and Tbx21-1- Th-cellinduced colitis without monocyte depletion (Figure $\mathbf{6 g}, \mathbf{h}$ ).

In colon explant supernatants of monocyte-depleted mice vs. isotype control-treated animals, reduced protein levels of IFN- $\gamma$ were detected $(P=0.1$, Figure $6 \mathbf{i})$. Levels of IL-12p40 were significantly reduced in monocyte-depleted mice compared with isotype controls and were similar to those measured in Tb $221^{-1-}$ Th-cell-induced colitis (Figure 6i). Levels of IL-1 $\beta$ and tumor necrosis factor did not differ significantly between monocyte-depleted mice and isotype controls. Taken together, in established colitis, monocytes and macrophages stabilized Th-cell numbers in the inflamed tissue, but did not influence weight loss, diarrhea, or overall colon histopathology.

\section{Th-cell-derived IFN- $\gamma$ directs type 1 inflammation but is dispensable for the clinical manifestation of colitis}

As Tbx $21^{-1-}$ Th cells maintained the ability to express IFN $-\gamma$, we tested whether Th-cell-derived IFN- $\gamma$ is critically involved in colitis induction and transferred naive $\mathrm{WT}$ and Ifng ${ }^{-1-} \mathrm{Th}$ cells into $\mathrm{Rag}^{-1-}$ mice. IFN- $\gamma$-deficient Th cells caused colitis indistinguishable from that induced by WT Th cells concerning weight loss, diarrhea, and numbers of Th cells in colon, spleen, 

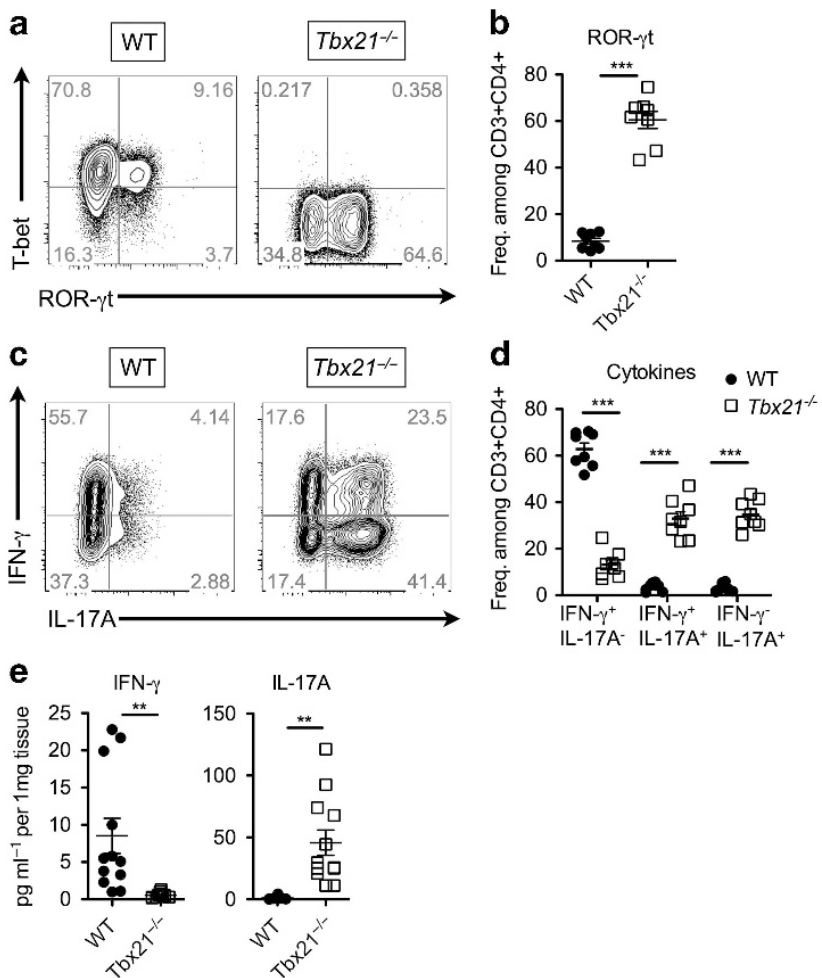

Figure 3 T-bet-deficient Th cells differentiate into Th1/17 cells. Naïve WT and Tbx21 $1^{-1-}$ CD4 + T cells were transferred into Rag ${ }^{-1}$ recipients. (a, b) Intracellular staining of T-bet and ROR- $\gamma$ t in colonic CD3 + CD4 + Th cells of colitic mice (day 16-21 after transfer). (c, d) Intracellular staining of IFN- $\gamma$ and IL-17A in colonic CD3 + CD4 + Th cells after $4 \mathrm{~h}$ PMA/ionomycin restimulation. Shown are $n=8$ mice per group from two out of more than five experiments with similar results. (e) IFN- $\gamma$ and IL-17A concentration in supernatants of $24 \mathrm{~h}$ colon explant cultures. Shown are $n=12$ mice per group from two out of four experiments with similar results. ${ }^{* \star} P<0.01$ and ${ }^{* * *} P<0.001$ by Student's $t$ test for independent samples. IFN, interferon; IL, interleukin; PMA, phorbol 12-myristate 13-acetate; WT, wild type.

and mesenteric lymph nodes (Figure $7 \mathbf{a}, \mathbf{b}$ and Supplementary Figure S7). However, the histopathology scores induced by Ifng $^{-1-}$ Th cells were reduced compared with WT Th cells (Figure $7 \mathbf{c}, \mathbf{d}$ ). To address the relevance of Th-cell-derived IFN- $\gamma$ for the composition of the inflammatory infiltrate, we compared the numbers of colonic myeloid cells in colitis induced by WT or Ifng ${ }^{-1-}$ Th cells. While the numbers of eosinophils and neutrophils were similar between WT and Ifng $^{-1-}$ Th-cell-induced colitis, the numbers of monocytes and macrophages were significantly reduced in the colons of colitis induced by IFN- $\gamma$-deficient vs. WT Th cells (Figure 7e). Macrophages from colitis induced by Ifng ${ }^{-1-}$ Th cells expressed increased levels of Arg1 and decreased levels of Fpr1 and Nos2 compared with macrophages from WT Th-cell-induced colitis (Figure 7f) indicating that IFN- $\gamma$ expressed by Th cells enhances M1 vs. M2 polarization of macrophages. Taken together, Th-cell-derived IFN- $\gamma$ controlled type 1 inflammation, i.e., attraction of monocytes and macrophages and their differentiation towards M1 but was dispensable for the clinical manifestation of colitis.

\section{Neutralization of IFN- $\gamma$ but not IL-17A abrogates colitis induced by WT and T-bet-deficient Th cells}

To interrogate the roles of the IFN- $\gamma$ and IL-17A in colitis induced by WT or T-bet-deficient Th cells, these cytokines were blocked by $1 \mathrm{mg}$ of neutralizing antibodies every 4 days beginning at the day of T-cell transfer. Neutralization of IFN- $\gamma$ completely prevented weight loss and colon histopathology, when compared with isotype control both in WT and Tbx21 $1^{-1-}$ Th-cell-induced colitis (Figure 8a-c, Supplementary Figure S8). Neutralization of IL-17A resulted in a trend towards higher weight loss compared with isotype control in WT $(P=0.054)$ and Tbx21 $1^{-1-}(P=0.27)$ Th-cell-induced colitis (Figure 8a). The colon histopathology score was increased by IL-17A neutralization compared with isotype control in WT $(P<0.05)$ and Tbx21 ${ }^{-1-}(P=0.09)$ Th-cell-induced colitis (day 12-15) (Figure 8b, c, Supplementary Figure S8). Thus, colitis induction by both WT and T-betdeficient Th cells requires IFN- $\gamma$ but is not dependent on IL-17A.

\section{DISCUSSION}

Here, we dissect the role of T-bet expression by Th cells for the induction and perpetuation of transfer colitis. T-bet expression by Th cells controls their survival and localization as well as the accumulation of monocytes and macrophages in the inflamed colon. It also enhances the differentiation of monocytes and macrophages towards the M1 phenotype. Conversely, monocytes/macrophages stabilize Th-cell numbers in the inflamed gut. T-bet-deficient Th cells efficiently induce colitis, as reflected by weight loss, diarrhea, and colon histopathology. They differentiate into Th1/17 cells, able to express both IFN- $\gamma$ and IL-17A. Antibody-mediated neutralization of IFN- $\gamma$ prevents the development of colitis. Neutralization of IL17A rather exacerbates colitis induced by WT and T-betdeficient Th cells demonstrating that colitis pathogenesis is not mediated by IL-17A. T-bet-deficient Th cells adapt the chemokine and chemokine receptor expression of Th17 cells, and promote the accumulation of M2 monocytes and macrophages as well as eosinophils in the inflamed colon. IFN- $\gamma$-deficient Th cells induce colitis comparable with T-betdeficient Th cells, suggesting that redundant sources of IFN- $\gamma$ can compensate for the deficiency of IFN- $\gamma$ production by Th cells. Overall, T-bet expressed by Th cells is an important nonredundant modulator of inflammation, but not a critical prerequisite of colitis induction by Th cells as such.

In the T-cell transfer-induced colitis model, naïve Th cells are transferred into syngenic, immunodeficient recipients where they, released from the control by $\mathrm{CD} 45 \mathrm{RB}^{\mathrm{low}} \mathrm{CD} 25+\mathrm{Th}$ cells, proliferate vigorously and react against the commensal microbiota. ${ }^{4,5}$ These Th cells differentiate into effector cells that cause wasting and diarrhea. Histopathology of the inflamed gut reveals epithelial hyperproliferation, goblet cell depletion, crypt abscess formation, and cellular infiltration. ${ }^{22}$ The cellular infiltrate consists of the inflammatory Th cells themselves, and myeloid cells invading the colon, especially inflammatory monocytes, macrophages, and neutrophils, ${ }^{23}$ and as we show here, eosinophils. 
a

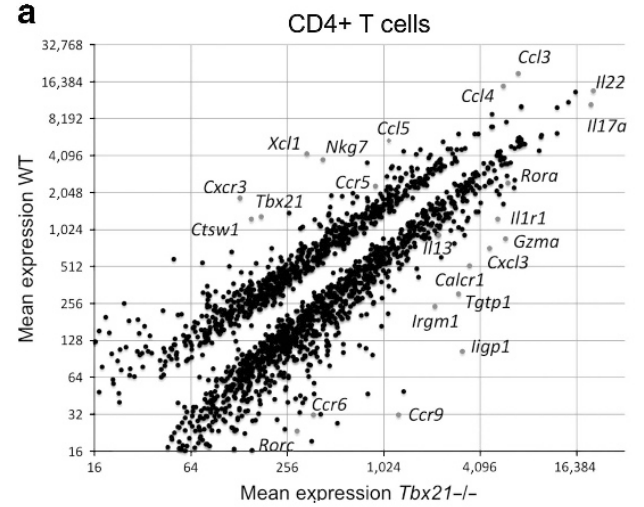

b

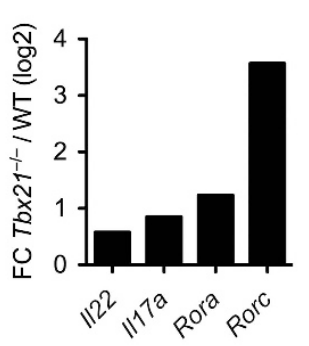

C

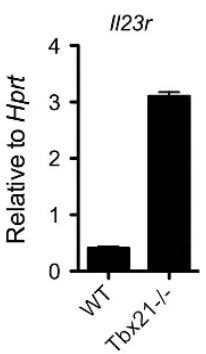

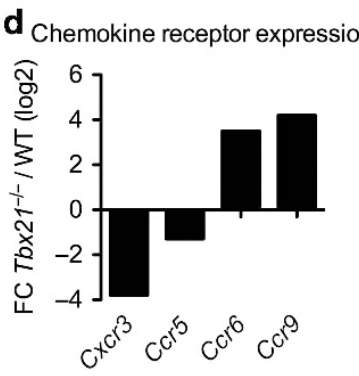

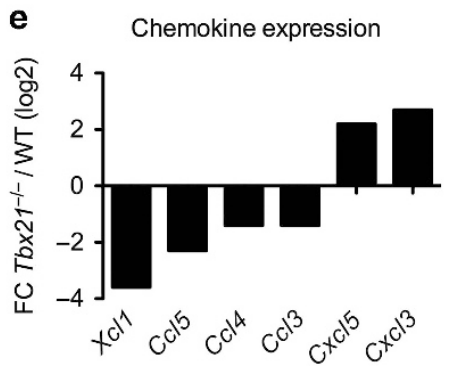

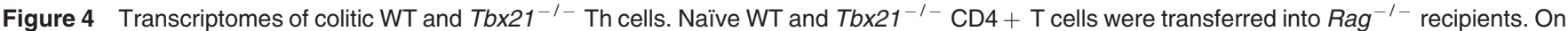
day 15, colonic CD3 + CD4 + Th cells from colitic mice were sorted by FACS and restimulated for $3 \mathrm{~h}$ with PMA/ionomycin. Isolated RNA was applied to microarrays. (a) Mean expression values of differentially expressed genes for WT vs. Tbx21 $1^{-1-}$ Th cells. (b) Log2 of FC of Th17-related cytokines and transcription factors in Tbx21-1- Th cells vs. WT. (c) II23r expression of colonic WT or Tbx21-1- CD4 + T cells as determined by quantitative real time PCR. (d, e) FC in expression of selected chemokine receptors (d) and chemokines (e) of colonic Tbx21 ${ }^{-l-}$ Th cells vs. WT. FACS, fluorescence-activated cell sorting; FC, fold change; PMA, phorbol 12-myristate 13-acetate; WT, wild type.

We show for the first time on the level of individual Th cells, by immunofluorescence staining, that essentially all Th cells in established colitis express high levels of the transcription factor T-bet. T-bet has been identified and characterized as the lineage transcription factor of Th1 cells, because it is both necessary and sufficient to induce the differentiation of activated Th cells into Th1 cells. ${ }^{7-9}$ Neurath and colleagues ${ }^{6}$ have described earlier that T-bet-deficient Th cells cannot induce colitis, when transferred into Rag-deficient or SCID mice, suggesting a critical, non-redundant role for T-bet in colitis induction. Here, we show, however, that T-bet-deficient Th cells of the C57BL/6 genetic background efficiently induce colitis, when transferred into Rag-deficient C57BL/6 mice. It can only be speculated that these contradictory results might be due to differences in the composition of the intestinal microbiota. No information on this is available for the mice analyzed by Neurath and colleagues. In the study presented here, mice were colonized with the murine commensals SFB and $H$. hepaticus to standardize the intestinal microbiota for the presence of these two colitogenic bacterial species. Both, SFB and H. hepaticus have been shown to enhance T-cell-induced colitis. ${ }^{17,19}$ Apart from these two types of colitogenic bacteria, added intentionally, mice receiving mutant and WT T cells were cohoused, to harmonize their microflora, and we have determined the overall composition of the microbiota of the colitic mice before and during development of colitis by $16 \mathrm{~s}$ rDNA sequencing (Zimmermann et al., manuscript submitted).
In contrast to Neurath et al., Gökmen and colleagues ${ }^{14}$ have demonstrated that T-bet-deficient Th cells are able to induce colitis with enhanced wasting and colon thickening. Here, we describe a third phenotype, namely colitis with significantly reduced histologic scores but unchanged weight loss and macroscopic evidence of colitis. Again, this could not only be due to differences in the microbiota, but also due to differences in the genetic setup of the experiment. While Gökmen et al. used Rag2 ${ }^{-1-}$ mice on Balb/c background, we used Rag1 ${ }^{-1-}$ mice on C57BL/6 background. In addition, Gökmen et al. did not analyze the intestinal histology in their colitic mice, and would have missed the differences that we describe here.

In the transfer colitis analyzed here, T-bet promotes the accumulation of Th cells not only in the inflamed colon, but also in secondary lymphoid organs. While T-bet does not influence the frequencies of Th cells actively proliferating, as determined by Ki-67 staining, it does influence the ratio of Bim to Bcl-2 in favor of Bcl-2, predictably supporting survival of the Th cells. ${ }^{20}$ We recently described that the transcription factors T-bet and Twist1, the latter being a hallmark of repeatedly reactivated Th1 cells, induce the expression of the microRNA-148a, which targets and suppresses Bim expression, and enhances the survival of repeatedly reactivated Th1 cells. ${ }^{24}$ Indeed, in the colitis analyzed here, T-bet-deficient Th cells from the inflamed colons underwent apoptosis at an increased rate, as compared with WT Th cells, when restimulated ex vivo. 

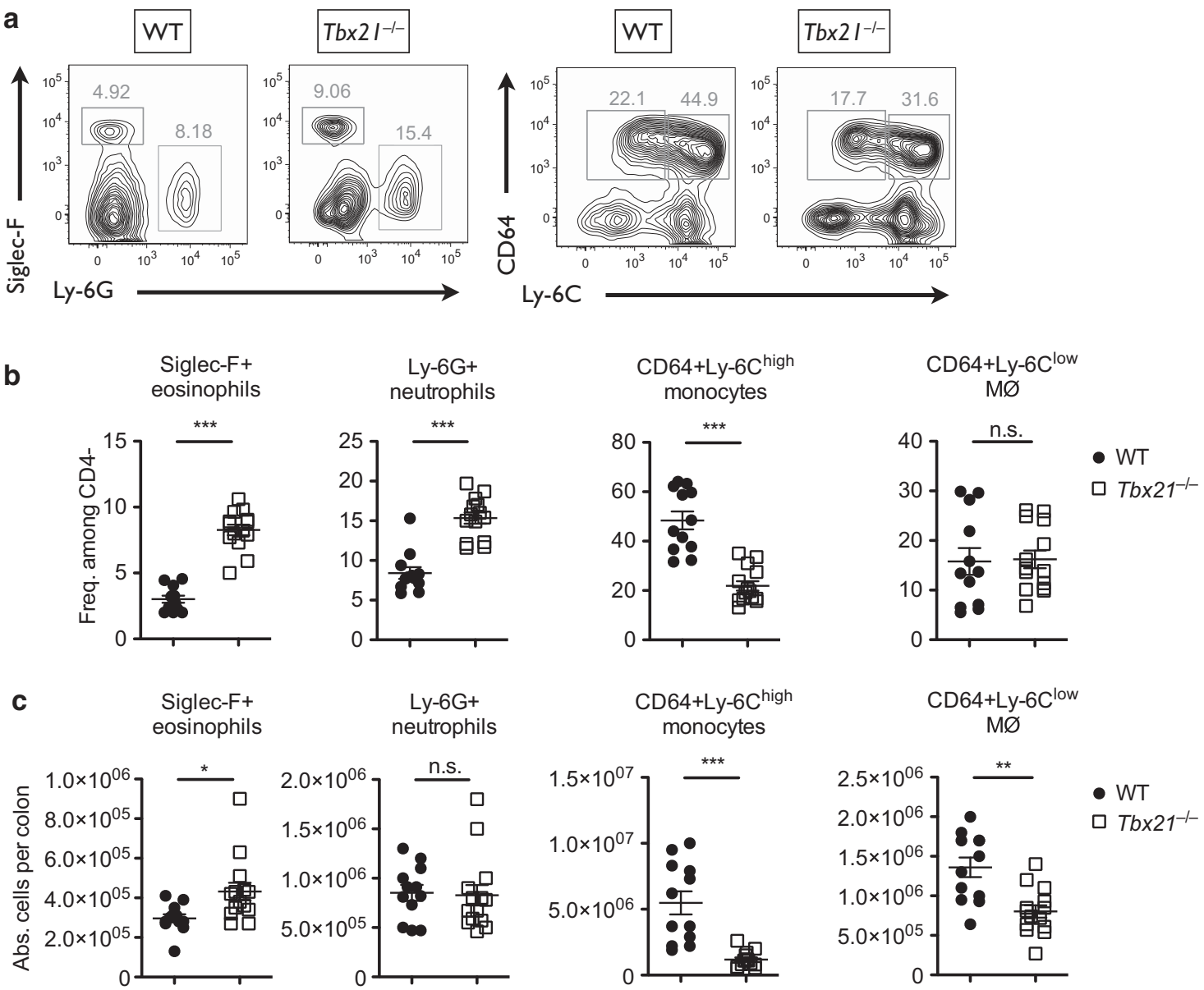

Figure 5 T-bet directs monocyte/macrophage accumulation in the inflamed colon. Naïve WT and Tbx21-1- CD4 + T cells were transferred into Rag ${ }^{-1-}$ recipients. (a) Identification of eosinophils (Siglec-F + ), neutrophils (Ly-6G + ), monocytes (CD64 + Ly-6C $\left.{ }^{\text {hi }}\right)$, and macrophages $(C D 64+$ Ly-6Clow $)$ among colonic CD4 - cells from colitic mice (day 16-21 after transfer). (b) Frequencies of subsets among CD4 - cells. (c) Absolute numbers of subsets per inflamed colon. Shown are $n=12-14$ mice per group from three out of more than five experiments with similar results with ${ }^{*} P<0.05$, ${ }^{\star *} P<0.01$, ${ }^{* * *} P<0.001$ by Student's $t$ test for independent samples. WT, wild type.

Moreover, T-bet is required for the accumulation of Th cells in the inflamed colon, whereas it prevents their accumulation in the small intestine, which may be because of the control of chemokine receptor expression by T-bet and/or ROR- $\gamma$ t. As we show here, T-bet-deficient $\mathrm{Th}$ cells recovered from the inflamed colon have significantly reduced expression of CXCR3. It was shown before that CXCR3 expression by Th cells is controlled directly by T-bet, and promotes immigration of Th cells into inflamed tissues. ${ }^{10}$ Conversely, in the absence of T-bet, Th cells express higher levels of CCR6 and CCR9, equipping them with the address code for homing to the small intestine. ${ }^{21}$ This may be due to the enhanced expression of ROR- $\gamma$ t by the T-bet-deficient colitis-inducing Th cells, as described here. In addition to CCR6, expression of IL-17A and IL-22, shown to be direct targets of ROR- $\gamma \mathrm{t}$, indicate the enhanced Th17 differentiation of T-bet-deficient colitic Th cells. ${ }^{25,26}$ These findings substantiate earlier studies reporting increased Th17 differentiation by T-bet-deficient compared with WT Th cells ${ }^{27-29}$ and confirm an inhibitory role for T-bet in Th17 differentiation. ${ }^{14,30}$

The direct effects of colitis-inducing Th cells on the non-hematopoietic cells of the colon, e.g., colonic epithelial cells, are not well defined. ${ }^{31}$ Here, in the colitis induced by T-bet-deficient vs. -sufficient Th cells, we do not observe quantitative differences in epithelial cell hyperproliferation. We show that T-bet-deficient colitic Th cells, as compared with WT Th cells, express higher levels of IL-17A, IL-17F, and IL-22. Importantly, increased levels of IL-17A are not responsible for the colitis-inducing potential of T-bet-deficient Th cells but instead seem to alleviate colitis, as we have demonstrated here by antibody-mediated neutralization of IL-17A. These data corroborate two recent reports demonstrating the pivotal beneficial role of IL-17A for the integrity of the intestinal epithelial barrier. ${ }^{32,33}$ Thus, although the increased IL-17A expression by T-bet-deficient Th cells has been found to be pathogenic in allergen-induced airway inflammation ${ }^{29}$ and cardiac allograft rejection, ${ }^{28}$ it appears to be protective in colitis, underlining a context-specific mode of action of IL-17A.

T-bet-deficient Th cells express substantial amounts of IFN- $\gamma$, which is a remarkable finding, as it questions the essential role of T-bet for the control of IFN- $\gamma$, a hallmark of Th1 cells. Similar IFN- $\gamma$ expression by T-bet-deficient Th cells has been observed in Mycobacterium tuberculosis infection, experimental 


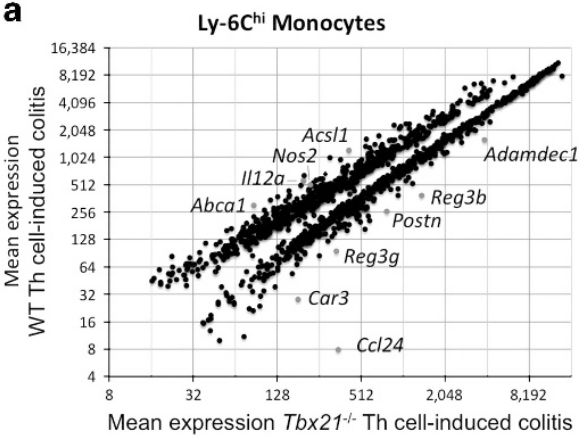

b

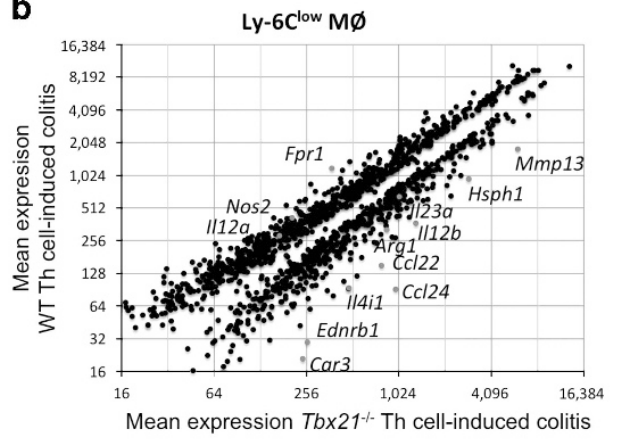

C

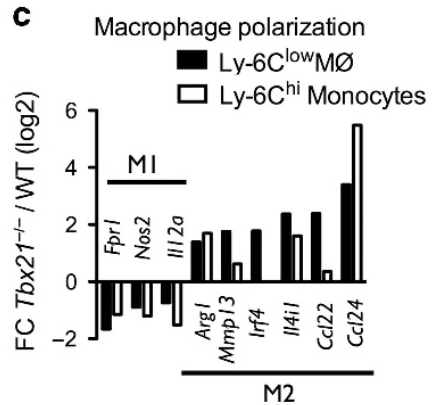

d

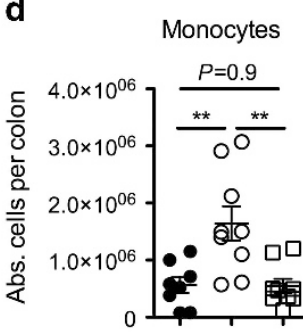

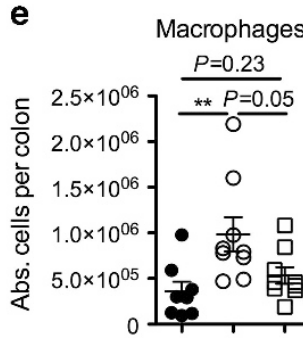
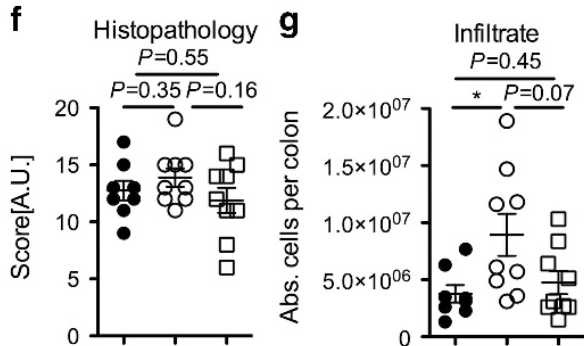

$\mathbf{h}$
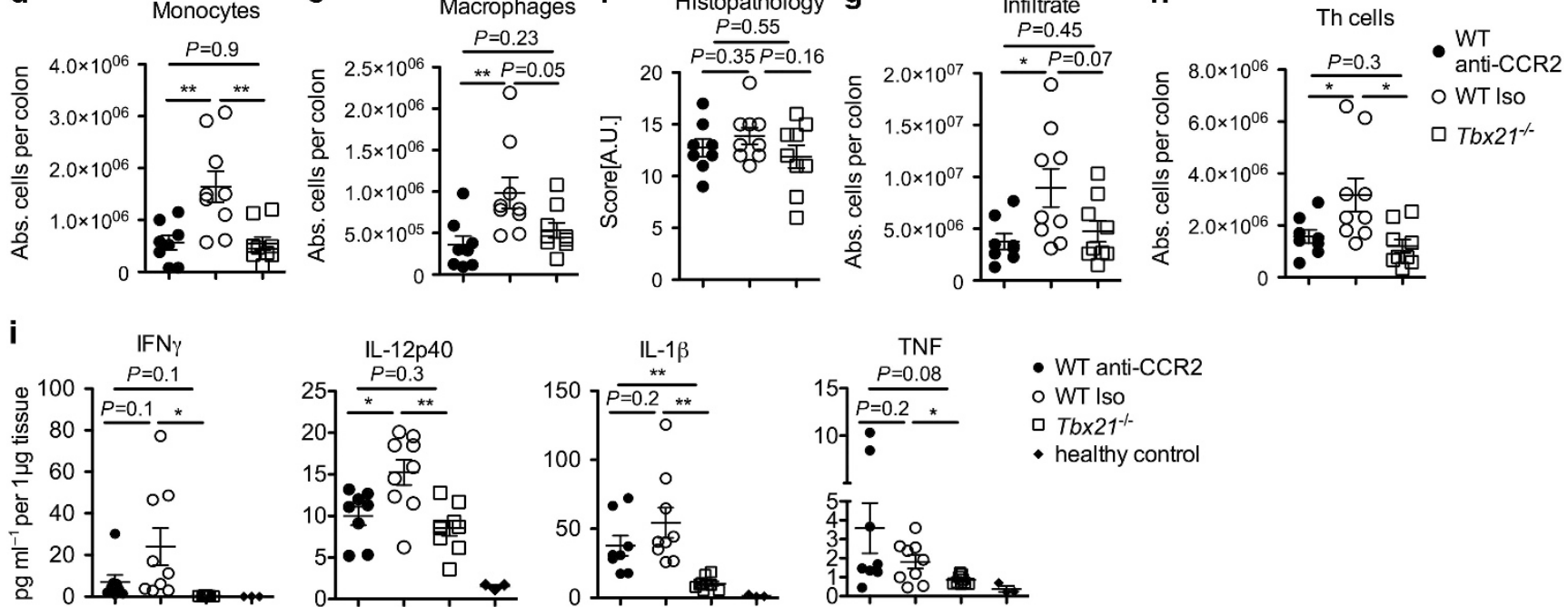

- WT anti-CCR2

○ WT Iso

Tbx21-1-

- healthy control

Figure 6 Phenotype and function of monocytes/macrophages in WT and Tbx21 ${ }^{-1-}$ Th-cell-induced colitis. Naïve WT and Tbx21 ${ }^{-1-}$ CD4 + T cells were transferred into $\mathrm{Rag}^{-\prime-}$ recipients. (a-c) Colonic CD64 + Ly-6C ${ }^{\text {hi }}$ monocytes and CD64 + Ly-6Clow macrophages were sorted by FACS when signs of colitis became apparent (day 16-21 after transfer). Isolated RNA was applied to microarrays. Mean gene expression levels of (a) monocytes and (b) macrophages from Tbx21 $1^{-I}$ Th-cell-induced colitis ( $x$ axis) vs. WT (yaxis). (c) Signature genes of M1 and M2 macrophage polarization depicted as FC of expression in Tbx21 $1^{-1}$ Th-cell-induced colitis vs. WT. (d-i) Starting on day 7 after colitis induction, monocytes were depleted in the WT group by daily i.p. injections of $20 \mu \mathrm{g}$ anti-CCR2 antibody vs. isotype control (iso). (d, e) Absolute numbers of monocytes (d) and macrophages (e) per colon. (f) Colon histopathology score. $(\mathbf{g}, \mathbf{h})$ Absolute number of infiltrating leukocytes $(\mathbf{g})$ per colon determined by flow cytometry according to leukocyte appearance (FSC/SSC) and Th cells (h). (i) Protein concentrations in the supernatant of colon explants cultured for $24 \mathrm{~h}$. (d-i) $n=8-9$ mice per group from two independent experiments with ${ }^{\star} P<0.05,{ }^{* *} P<0.01$ determined by Student's $t$ test for independent samples. FACS, fluorescence-activated cell sorting; FC, fold change; FSC, forward scatter; i.p., intraperitoneal; SSC, side scatter; WT, wild type.

autoimmune encephalomyelitis, allograft rejection, and in vitro Th-cell polarization. ${ }^{27,34-36}$ In $\mathrm{CD} 8+\mathrm{T}$ cells, the T-box transcription factor Eomesodermin (Eomes) can drive IFN- $\gamma$ expression in the absence of T-bet. ${ }^{37} \mathrm{We}$ detected Eomesexpressing WT and T-bet-deficient Th cells, but their frequencies were at least four times lower compared with the frequencies of IFN- $\gamma$-expressing Th cells (data not shown) suggesting that Eomes is not a major driver of T-bet-independent IFN- $\gamma$ expression. In Th17 cells, it has been shown that Runx1 or Runx3 can drive the expression of IFN- $\gamma$ independently of T-bet. ${ }^{27,38}$ Here, we have observed unimpaired expression of Runx1 and Runx3 by T-betdeficient Th cells providing a possible mechanism for how IFN- $\gamma$ expression could be driven in the absence of T-bet.

IFN- $\gamma$ has been reported to promote colon inflammation, as demonstrated by the use of antagonistic antibodies. ${ }^{12}$ However, IFN- $\gamma$-deficient Th cells have been described to efficiently induce colitis, suggesting that Th-cell-derived IFN- $\gamma$ is dispensable for the inflammation. ${ }^{13,39}$ Here, we have addressed this apparent contradiction: T-bet and IFN- $\gamma$ expression by Th cells are essential for the orchestration of type 1 inflammation. In the absence of T-bet, colitis could still be induced, but now was characterized by type 17 inflammation, i.e., abundance of Th17 cells, decreased infiltration of monocytes/macrophages, their polarization to M2 phenotype, and increased numbers of eosinophils. Still, this colitis induced by T-bet-deficient Th cells could completely be abrogated by the neutralization of IFN- $\gamma$ suggesting that IFN- $\gamma$ from T-bet-deficient Th1/17 cells contributes to colitis induction, but does not suffice to induce a type 1 inflammation. Our data further indicate that non-T-cell sources of IFN- $\gamma$ cannot replace Th-cell-derived IFN- $\gamma$ to induce a type 1 inflammation, but are sufficient to induce colitis as such. It has been shown for innate lymphoid cells that they could serve as alternative sources of IFN- $\gamma$ driving the development of colitis. ${ }^{40,41}$ We speculate that localization and cellular interaction of the IFN- $\gamma$-expressing cells determine the function of IFN- $\gamma$ of different cellular sources in the pathogenesis 

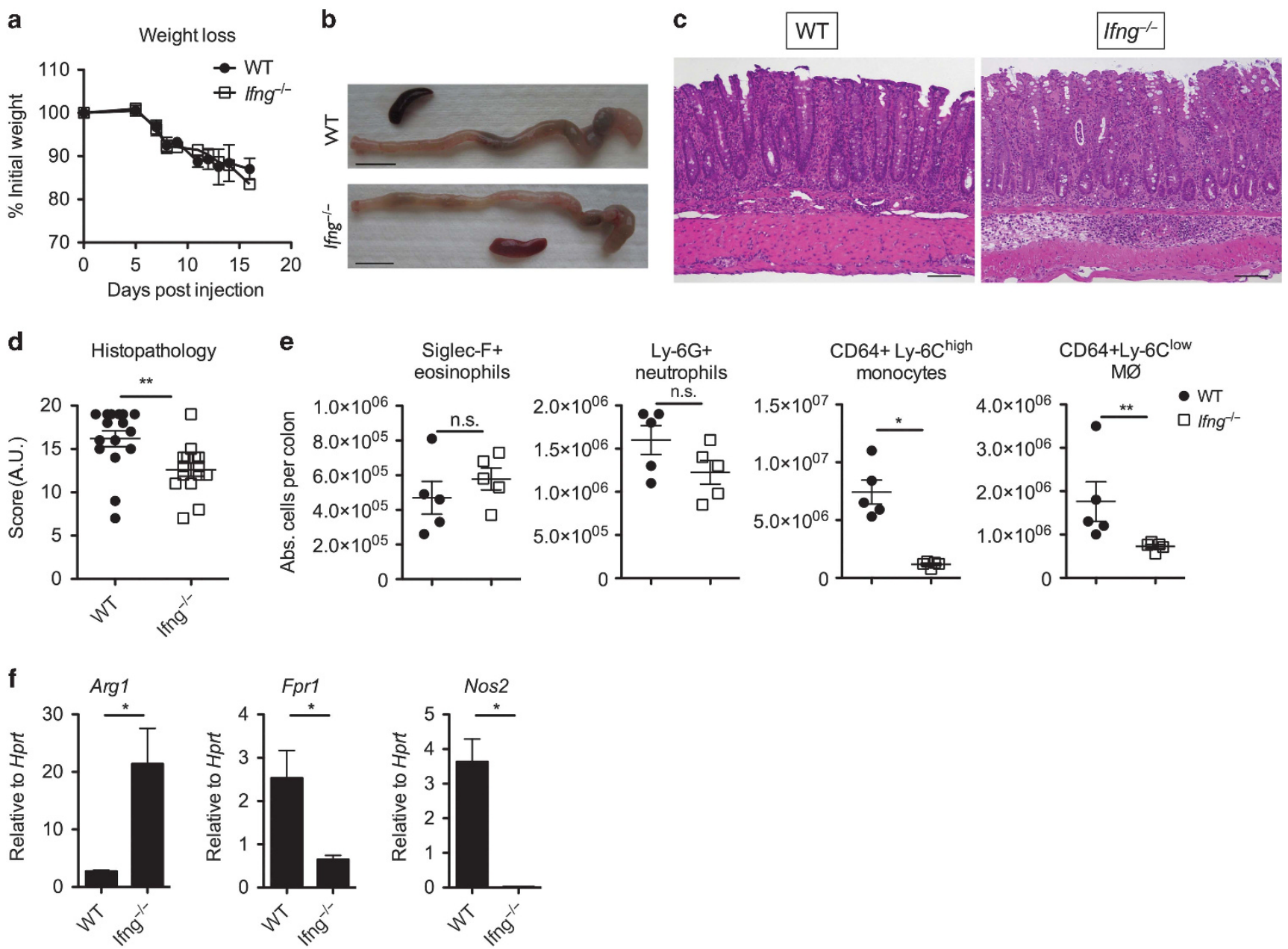

Figure 7 Th-cell-derived IFN- $\gamma$ directs monocyte and macrophage attraction and differentiation. Naïve WT and lfng ${ }^{-1-}$ CD $4+$ T cells were transferred into $\mathrm{Rag}^{-1-}$ recipients. (a) Weight loss relative to starting weight for $n=4$ mice per group from one out of three independent experiments.

(b) Macroscopic pictures of colons, scale bars are $10 \mathrm{~mm}$. (c) H\&E stainings of colons, scale bars are $100 \mu \mathrm{m}$. (d) Histopathology score for $n=14-16 \mathrm{mice}$ per group from three independent experiments. ${ }^{* \star} P<0.01$, ${ }^{\star \star \star} P<0.001$ determined by Student's $t$ test for independent samples. (e) Absolute numbers of myeloid cell subsets in colons of colitic mice (day 14) determined by flow cytometry. (f) On day 14, CD64 + Ly-6C low macrophages were sorted by FACS and gene expression was analyzed by qPCR. (e) and (f) show $n=5$ mice per group with ${ }^{*} P<0.05$ and ${ }^{*} P<0.01$ by Mann-Whitney test for independent samples. FACS, fluorescence-activated cell sorting; H\&E, hematoxylin and eosin; IFN, interferon; qPCR, quantitative PCR; WT, wild type.

of colitis. It is, thus, possible that whereas T-cell-derived IFN- $\gamma$ is critical for monocyte/macrophage accumulation and function, IFN- $\gamma$ provided by non-T cells may be required to induce pathogenic function in the transferred Th cells.

It has been suggested that the inflammation in transfer colitis is critically dependent on inflammatory myeloid cells recruited and activated by the colitogenic Th cells. ${ }^{42}$ We show here that the infiltration of the colon with inflammatory monocytes and macrophages is dependent on T-bet or IFN- $\gamma$ expression by the colitic Th cells. The observation that the numbers of monocytes and macrophages in the spleen are comparable between colitis induced by T-bet-deficient and WT Th cells suggests that T-bet specifically regulates the accumulation of monocytes and macrophages in the colon. We speculate that this might be due to the expression of CCR 1 and CCR 5 by monocytes and macrophages combined with T-bet-dependent expression of the corresponding ligands CCL3, CCL4, and CCL5 by the Th cells.
T-bet and IFN- $\gamma$ expression by colitic Th cells also affects the phenotype of monocytes and macrophages in the colon. Their transcriptome resembles that of M1 macrophages. ${ }^{43}$ In colitis induced by not only T-bet-deficient but also IFN- $\gamma$-deficient Th cells, monocytes and macrophages express a transcriptome of the M2 type, likely because of the decreased levels of IFN- $\gamma$ in the direct communication between Th cells and monocyte/ macrophages. This includes expression of CCL24. CCL24 is a ligand of CCR3, a chemokine receptor that in colitis is expressed by eosinophils, but not by Th cells, neutrophils, or monocytes/macrophages (data not shown). CCL24 expression by $\mathrm{M} 2$ monocytes and macrophages could thus explain the increased recruitment of eosinophils to the inflamed colon in colitis induced by T-bet-deficient Th cells. Despite these differences in the cellular composition of the leukocyte infiltrate, the clinical parameters of colitis, weight loss, and diarrhea are similar in colitis induced by T-bet-deficient vs. T-bet-sufficient Th cells. In terms of histopathology, 


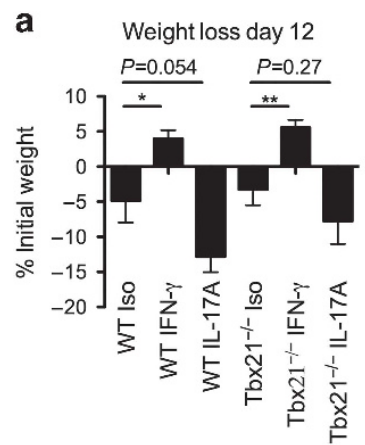

b

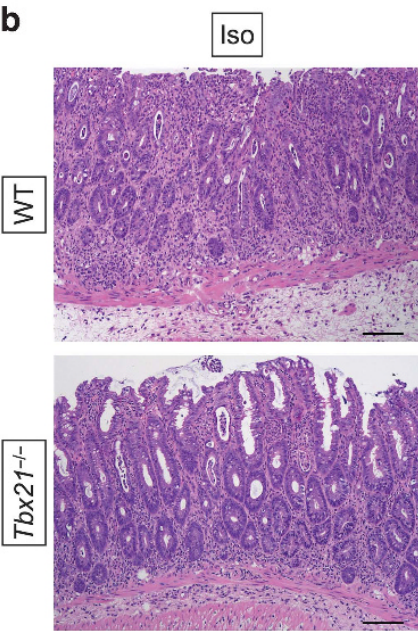

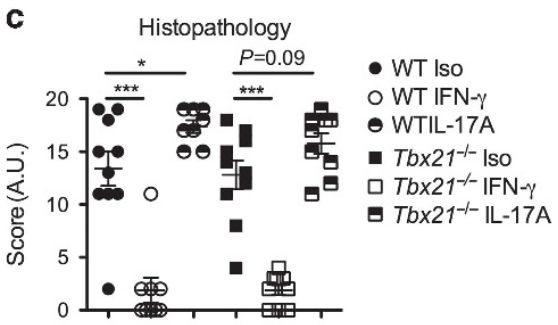
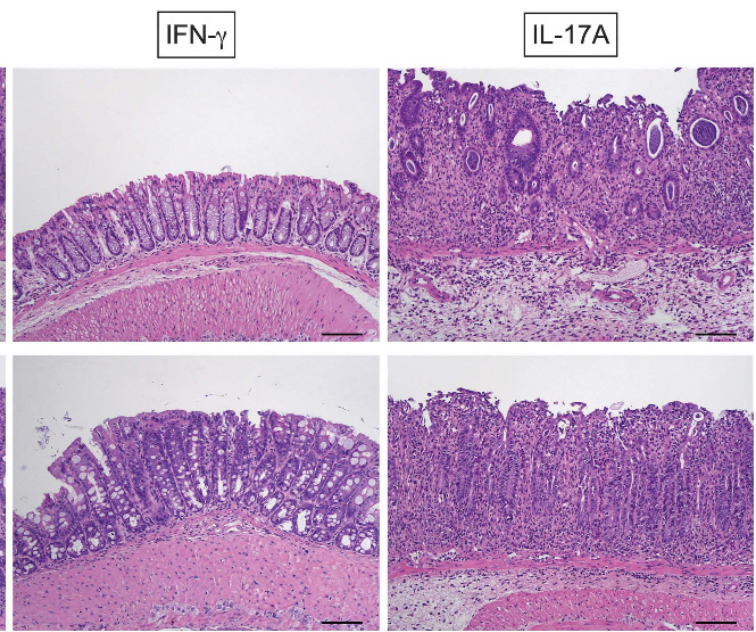

Figure 8 Neutralization of IFN- $\gamma$ but not IL-17A attenuates colitis. Naïve WT and Tbx21 ${ }^{-1}$ CD4 + T cells were transferred into Rag ${ }^{-1-}$ recipients and $1 \mathrm{mg}$ anti-IFN- $\gamma$, anti-IL-17A antibody or isotype control were injected i.p. every 4 days beginning at day 0 . (a) Weight loss relative to initial weight. (b) H\&E stainings of colons upon analysis (day 12-15), scale bars are $100 \mu \mathrm{m}$. (c) Colon histopathology score. $n=8-10$ mice per group from two independent experiments with ${ }^{\star} P<0.05$, ${ }^{\star \star} P<0.01$, and ${ }^{* \star *} P<0.001$ determined by Student's $t$ test for independent samples. H\&E, hematoxylin and eosin; IFN, interferon; IL, interleukin; i.p., intraperitoneal; WT, wild type.

however, crypt abscesses, goblet cell loss, and cellular infiltration are reduced in colitis induced by T-bet-deficient Th cells.

Depletion of monocytes/macrophages in established colitis, induced by WT Th cells results in a significant reduction in the number of infiltrating leukocytes and Th cells. However, weight loss, diarrhea, and colon histopathology are not affected. The reduction in numbers of infiltrating Th cells suggests that $\mathrm{T}$-bet expression by Th cells is part of a positive feedback loop between monocytes/macrophages and Th cells. While on one hand, T-bet controls the expression of CXCR3 and monocytes/ macrophages express the corresponding ligands, CXCL10 and CXCL11, T-bet on the other hand induces expression of the chemokines CCL3, CCL4, and CCL5 in Th cells, which are capable of attracting CCR1- and CCR5-bearing monocytes/ macrophages. Moreover, monocytes/macrophages express the cytokine subunits IL-12p40 and IL-12p35, enhancing T-bet expression and imprinting Th1 differentiation. ${ }^{9}$ Depletion of monocytes/macrophages significantly reduces the concentration of IL-12p40 in the colon. However, the amounts of the colitogenic, inflammatory cytokines IL-1 $\beta$ and tumor necrosis factor, ${ }^{44,45}$ in the colon do not change upon monocyte depletion. These cytokines are also expressed by granulocytes of the colon, whereas IL-12p40 is not (data not shown).

Transfer colitis is thought to model the unleashing of Th cells from physiological control, allowing them to interact with the intestinal microbiota. The phenotype of Th cells from the colon of mice with transfer colitis and of humans with $\mathrm{CD}$ is similar, in that Th cells expressing IFN- $\gamma$ and/or IL-17A are prevailing. ${ }^{25,46}$ It was described earlier, that T-bet-deficient Th cells cannot induce transfer colitis. ${ }^{6}$ In human $\mathrm{CD}$, high expression of the gene TWIST1 suggests that Th cells from the inflamed colon have a history of repeated restimulation in the presence of IL-12, i.e., as Th1 cells. ${ }^{47}$ However, as we demonstrate here, the clinical symptoms of colitis can be induced independently of T-bet or IFN- $\gamma$ expression by Th cells. T-bet- and IFN- $\gamma$-deficient Th cells induce colitis characterized by reduced monocyte/macrophage accumulation in the colon, M2 instead of M1 differentiation and, in the case of T-bet-deficient Th cells, increased abundance of eosinophils.

\section{METHODS}

Mice. C57BL6/J, Rag1-1-,$T b \times 21^{-1-}$ and $I f n g^{-/-}$mice were housed and bred under specific pathogen-free conditions in individually ventilated cages. Two weeks prior to T-cell transfer, Rag1 ${ }^{-/-}$ 
Table1 Histopathology score

\begin{tabular}{|c|c|c|c|c|c|c|}
\hline & 0 & 1 & 2 & 3 & 4 & 5 \\
\hline Mucosal infiltration & None & Mild & Moderate & Severe & & \\
\hline Submucosal Infiltration & None & Minimal, focal & Mild & Moderate, diffuse & Severe & Transmural \\
\hline Epithelial hyperplasia & None & Mild & Moderate & Severe & & \\
\hline Goblet cell loss & None & Mild & Moderate & Severe & & \\
\hline Crypt abscesses & None & Sporadic & Few & Many & Crypt loss & \\
\hline Area affected & None & $<25 \%$ & $<50 \%$ & $>50 \%$ & & \\
\hline
\end{tabular}

were colonized by oral gavage with a fecal bacteria suspension containing SFB and Helicobacter species (H. spp). Successful colonization was confirmed by PCR from fecal DNA with the following primer pairs: H. spp Fw: $5^{\prime}$-ctatgacgggtatccggc- $3^{\prime}, \mathrm{Rv}$ : $5^{\prime}$-attccacctacctctccca-3', SFB Fw: $5^{\prime}$-gacgctgaggcatgagagcat-3', Rv: $5^{\prime}$-gacggcacggattgttattca- $3^{\prime}$. Presence of $H$. hepaticus was determined with Fw: $5^{\prime}$-gcatttgaaactgttactctg-3', Rv: 5'-ctgttttcaagctcccc-3'. Mice were handled in accordance with good animal practice as defined by the German animal welfare bodies. All experiments were approved by the regulatory office Landesamt für Gesundheit und Soziales' in Berlin, Germany.

Colitis induction. Colitis was induced as published before. ${ }^{4}$ Briefly, $\mathrm{CD} 4+\mathrm{T}$ cells from spleen and $\mathrm{pLN}$ of C57BL/6J donors were purified by high-gradient magnetic cell sorting (MACS) using mouse CD4 direct beads (L3T4, Miltenyi Biotec, Bergisch Gladbach, Germany). Viable $\mathrm{CD} 4{ }^{+} \mathrm{CD} 45 \mathrm{RB}^{\mathrm{hi}} \mathrm{CD} 25^{-}$cells were isolated by fluorescenceactivated cell sorting (FACS) with a FACSAria I (BD Biosciences, Heidelberg, Germany). Cells $\left(4 \times 10^{5}\right)$ were injected i.p. or intravenous into each $R a g 1^{-1-}$ recipient. Different experimental groups were cohoused in the same cages. Mice were killed 2-3 weeks after transfer, when signs of diarrhea and weight loss became apparent. Recipient mice that upon analysis did not have any detectable T cells because of failure of the adoptive T-cell transfer were excluded from the analysis.

In vivo antibody application. Monocytes were depleted beginning from day 7, when mice showed first clinical signs of colitis (weight loss and diarrhea) by daily i.p. injection of $20 \mu \mathrm{g}$ anti mouse CCR2 (MC21) or isotype control as published before. ${ }^{48}$ Cytokines were neutralized by i.p. injection of $1 \mathrm{mg}$ anti IL-17A (TC11-18H10.1, Biolegend, Fell, Germany), anti-IFN- $\gamma$ (R4.6A2, DRFZ hybridoma, Berlin, Germany), or isotype control (anti-mouse kappa chain, 187.1, DRFZ hybridoma) every 4 days beginning at the day of Th-cell transfer.

Histology. Colons were fixed in $4 \%$ paraformaldehyde at $4{ }^{\circ} \mathrm{C}$ in the "Swiss roll" formation overnight. After washing with phosphatebuffered saline and dewatering, colons were embedded in paraffin. Sections were stained with hematoxylin and eosin. Colitis histopathology was scored in a blinded manner according to an adapted version of a score published elsewhere ${ }^{22}$ (see Table 1). Score values for single parameters were summed up to a total score ranging to a maximum of 21 .

Isolation of lamina propria leukocytes. Lamina propria leukocytes were isolated from colon and small intestine as described before. ${ }^{49}$ In brief, intestines were freed from fat, opened longitudinally, and washed with phosphate-buffered saline. The epithelial layer was stripped off in two rounds of incubation in calcium/magnesium-free Hank's balanced salt solution with $5 \mathrm{mM} \mathrm{EDTA}$ and $10 \mathrm{mM}$ HEPES for $20 \mathrm{~min}$ at $37^{\circ} \mathrm{C}$. To obtain a single cell suspension of the mucosa, intestines were minced into small pieces and incubated three times for $20 \mathrm{~min}$ at $37^{\circ} \mathrm{C}$ with $0.5 \mathrm{mg} \mathrm{ml}^{-1}$ Collagenase D (Roche, Mannheim, Germany), $0.5 \mathrm{mg} \mathrm{ml}^{-1}$ DNase I (Sigma-Aldrich, Hamburg, Germany) and
$0.5 \mathrm{U} \mathrm{ml}^{-1}$ Dispase (BD Biosciences). For small intestines, lamina propria leukocytes were separated from debris by centrifugation over a Percoll gradient.

Colon explant supernatants and cytometric bead assay. After washing, a 5-mm piece from the middle of each colon was incubated for $24 \mathrm{~h}$ in RPMI with $10 \%$ fetal calf serum. Protein amounts of various cytokines were determined in the supernatant with the Bio-Plex system (Bio-Rad, München, Germany) following the manufacturer's instructions and normalized to the weight of the colon explant.

Restimulation and flow cytometry. For intracellular cytokine staining, cells were restimulated with $10 \mathrm{ng} \mathrm{ml}^{-1} \mathrm{PMA}$ and $1 \mu \mathrm{g} \mathrm{ml}^{-1}$ ionomycin in Iscove's modified Dulbecco's medium, containing $10 \%$ fetal calf serum at $5 \times 10^{6}-1 \times 10^{7}$ cells ml ${ }^{-1}$ for a total of 4 h. $^{50}$ After $1 \mathrm{~h}$, brefeldin A was added to a final concentration of $5 \mu \mathrm{g} \mathrm{ml}^{-1}$. Cells were stained with a fixable live/dead discrimination dye for $20 \mathrm{~min}$ on ice, fixed with Cytofix/Cytoperm buffer (BD) and stained in $0.5 \% \mathrm{w} / \mathrm{v}$ Saponin for $20 \mathrm{~min}$ on ice. For transcription factor staining, cells were stained with the fixable live/dead discrimination dye directly ex vivo, fixed with the Foxp3 staining buffer kit (eBioscience, Frankfurt am Main, Germany) for $1 \mathrm{~h}$ on ice and stained in the $1 \times$ perm buffer from the Foxp3 staining buffer kit for $1 \mathrm{~h}$ on ice. Samples were acquired on a MACS Quant (Miltenyi Biotec) or a FACSCantoII (BD Biosciences). The following antibodies and reagents were used (clone, supplier): Annexin-V PE (Biolegend), Bcl-2 PE (3F11, BD Biosciences), Bim Alexa Fluor 647 (14A8, Merck Millipore, Darmstadt, Germany), CD3 APC-eFluor 780 (145-2C11, eBioscience), CD4 Pe-Cy7 (RM4-5, eBioscience), CD45RB PE (16A, BD Biosciences), CD25 APC (PC61, Biolegend), CD64 APC (X54-5/7.1, eBioscience), F4/80 Pe-Cy7 (BM8, Biolegend), Foxp3 eFluor 450 (FJK-16 s, eBioscience), GATA-3 Alexa Fluor 488 (L50-823, BD Biosciences), GM-CSF PerCP-Cy5.5 (MP122E9, Biolegend), IFN- $\gamma$ PerCP-Cy5.5 (XMG1.2, eBioscience), IL-4 PE (BVD4-1D11, Miltenyi Biotec), IL-10 PE (JES5-16E3, eBioscience), IL-13 PE (eBio13A, eBioscience), IL-17A FITC (TC11-18H10.1, Biolegend), IL-17F Alexa Fluor 488 (eBio18F10, eBioscience), IL-22 APC (IL22JOP, eBioscience), Ki-67 Alexa Fluor 488 (B56, eBioscience), Ly-6C Pacific Blue (HK1.4, Biolegend), Ly-6G PerCPCy5.5 (1A8, Biolegend), MHC-II APC-Cy7 (M5/114.15.2, Biolegend), ROR- $\gamma$ t PE (Q31-378, BD Biosciences), Siglec-F PE (E50-2440, BD Biosciences), T-bet Alexa Fluor 647 (4B10, Biolegend), TNF Pacific Blue (MP6-XT22, own conjugate).

Cell sorting, microarray analysis, and quantitative PCR. Th cells $(\mathrm{CD} 3+\mathrm{CD} 4+)$, monocytes (CD4 - CD64 + Ly-6C hi), and macrophages $\left(\mathrm{CD} 4-\mathrm{CD} 64+\mathrm{Ly}-6 \mathrm{C}^{\mathrm{lo}}\right)$ were sorted from colon lamina propria leukocytes (FACSAria I, BD Biosciences). Purified RNA was hybridized to MG 430_2 GeneChips (Affymetrix, High Wycombe, UK) according to the manufacturer's instructions. Significantly, differentially regulated genes were analyzed as described before. ${ }^{51}$ The chip data discussed in this publication have been deposited in NCBI's Gene Expression Omnibus and are accessible through GEO Series accession number GSE64696. For quantitative PCR analysis, RNA was 
reversely transcribed using the Taqmann Reverse Transcript Reagent Kit (Thermo Fisher Scientific, Schwerte, Germany) followed by quantitative PCR using SYBR green (Roche) and a StepOne Plus (Thermo Fisher Scientific). The following primer pairs were used:

Arg1 Fw: $5^{\prime}$-acaagacagggctcctttcag- $3^{\prime}$, Arg1 Rv: $5^{\prime}$-taaagccactgccgtgt tca-3',Fpr1 Fw: $5^{\prime}$-agttcaggagtctacaagatggac- $3^{\prime}$, Fpr1 Rv: $5^{\prime}$-ccagaacga tgtagccagca- $3^{\prime} H$ prt Fw: $5^{\prime}$-tcctcctcagaccgctttt- $3^{\prime}, H$ prt Rv: $5^{\prime}$-cataacctg gttcatcatcgc- $3^{\prime}$, Il $23 r$ Fw: $5^{\prime}$-ggcttttcggaacctcatgc- $3^{\prime}$, Il23r Rv: $5^{\prime}$-ccggtgg atgtccagaggaa- $3^{\prime}, N o s 2 \mathrm{Fw}: 5^{\prime}$-ccaccttggtgaagggactg- $3^{\prime}$, Nos2 Rv: $5^{\prime}$-caa cgttctccgttctcttgc- $3^{\prime}$.

Data presentation and statistical evaluation. Graphs depict mean \pm s.e.m. unless stated otherwise. Statistical analysis was carried out with Graph pad prism version 5 using the Mann-Whitney test for unpaired samples or the Student's $t$ test for unpaired samples depending on the group sizes.

SUPPLEMENTARY MATERIAL is linked to the online version of the paper at http://www.nature.com/mi

\section{ACKNOWLEDGMENTS}

We would like to thank F Siracusa, F Padberg, and U Hoffmann for experimental support and $\mathrm{H}$ Schliemann for the preparation of microarrays. We are grateful to $\mathrm{J}$ Kirsch and T Kaiser, operators of the flow cytometry core facility (FCCF). This work was supported by the German Research Council (DFG SFB633, SFB 650, and GRK1121) and the European Research Council advanced grant ERC-2010-AdG_20100317 Grant 268987. M-FM is supported by the "e:Bio Innovationswettbewerb Systembiologie" program of the Federal Ministry of Education. PM is supported by EUTRAIN, a FP7 Marie Curie Initial Training Network for Early Stage Researchers funded by the European Union. The DRFZ is a Leibniz Institute.

\section{AUTHOR'S CONTRIBUTIONS}

$\mathrm{JZ}$ designed and performed the research, analyzed the data, and wrote the paper. $\mathrm{H}-\mathrm{DC}$ and $\mathrm{AR}$ designed and supervised the research, analyzed the data, and edited the paper. JRG analyzed the microarray data. MM provided the anti-CCR2 antibody and edited the paper. AAK scored the histopathology. MW, JL, CH, RR, PM, KL, and KW performed experiments. $\mathrm{ML}$ and $\mathrm{M}-\mathrm{FM}$ advised the research and edited the paper.

\section{DISCLOSURE}

The authors declared no conflict of interest.

c) 2016 Society for Mucosal Immunology

\section{REFERENCES}

1. Saro, C. \& Aladrén, B.S. Inflammatory bowel diseases: a disease (s) of modern times? Is incidence still increasing? World J. Gastroenterol. 14, 5491 (2008).

2. Burt, R.K. et al. Autologous nonmyeloablative hematopoietic stem cell transplantation in patients with severe anti-TNF refractory Crohn disease: long-term follow-up. Blood 116, 6123-6132 (2010).

3. Stronkhorst, A. et al. CD4 antibody treatment in patients with active Crohn's disease: a phase 1 dose finding study. Gut 40, 320-327 (1997).

4. Powrie, F., Leach, M.W., Mauze, S., Caddle, L.B. \& Coffman, R.L. Phenotypically distinct subsets of CD4 + T cells induce or protect from chronic intestinal inflammation in C. B-17 scid mice. Int. Immunol. 5, 1461-1471 (1993).

5. Feng, T., Wang, L., Schoeb, T.R., Elson, C.O. \& Cong, Y. Microbiota innate stimulation is a prerequisite for $\mathrm{T}$ cell spontaneous proliferation and induction of experimental colitis. J. Exp. Med. 207, 1321-1332 (2010).

6. Neurath, M.F. et al. The transcription factor T-bet regulates mucosal Tcell activation in experimental colitis and Crohn's disease. J. Exp. Med. 195, 1129-1143 (2002).

7. Szabo, S.J. et al. A novel transcription factor, T-bet, directs Th1 lineage commitment. Cell 100, 655-669 (2000).
8. Szabo, S.J. Distinct effects of T-bet in $\mathrm{TH} 1$ lineage commitment and IFN-gamma production in CD4 and CD8 T cells. Science 295, 338-342 (2002).

9. Schulz, E.G., Mariani, L., Radbruch, A. \& Hofer, T. Sequential polarization and imprinting of type $1 \mathrm{~T}$ helper lymphocytes by interferon-\&gamma; and interleukin-12. Immunity 30, 673-683 (2009).

10. Lazarevic, V., Glimcher, L.H. \& Lord, G.M. T-bet: a bridge between innate and adaptive immunity. Nat. Rev. Immunol. 13, 1-13 (2013).

11. Fuss, I.J. et al. Disparate CD4 + lamina propria (LP) lymphokine secretion profiles in inflammatory bowel disease. Crohn's disease LP cells manifest increased secretion of IFN-gamma, whereas ulcerative colitis LP cells manifest increased secretion of IL-5. J. Immunol. 157, 1261-1270 (1996).

12. Powrie, F. et al. Inhibition of Th1 responses prevents inflammatory bowel disease in scid mice reconstituted with CD45RBhi CD4 + Tcells. Immunity 1, 553-562 (1994)

13. Simpson, S.J. et al. $\mathrm{T}$ cell-mediated pathology in two models of experimental colitis depends predominantly on the interleukin 12/Signal transducer and activator of transcription (Stat)-4 pathway, but is not conditional on interferon gamma expression by T cells. J. Exp. Med. 187, 1225-1234 (1998).

14. Gokmen, M.R. et al. Genome-Wide Regulatory Analysis Reveals That T-bet Controls Th17 Lineage Differentiation through Direct Suppression of IRF4. J. Immunol. 191, 5925-5932 (2013).

15. Leppkes, M. et al. ROR $\gamma$-expressing Th17 cells induce murine chronic intestinal inflammation via redundant effects of IL-17 A and IL-17F. Gastroenterology 136, 257-267 (2009).

16. Durant, L. et al. Diverse targets of the transcription factor STAT3 contribute to T cell pathogenicity and homeostasis. Immunity 32, 605-615 (2010).

17. Stepankova, R. et al. Segmented filamentous bacteria in a defined bacterial cocktail induce intestinal inflammation in SCID mice reconstituted with CD45RBhigh CD4 + T cells. Inflamm. Bowel Dis. 13, 1202-1211 (2007).

18. Ivanov, I.I. et al. Induction of intestinal Th17 cells by segmented filamentous bacteria. Cell 139, 485-498 (2009).

19. Cahill, R.J. et al. Inflammatory bowel disease: an immunity-mediated condition triggered by bacterial infection with Helicobacter hepaticus. Infect. Immun. 65, 3126-3131 (1997).

20. Wojciechowski, S. et al. Bim/Bcl-2 balance is critical for maintaining naive and memory T cell homeostasis. J. Exp. Med. 204, 1665-1675 (2007).

21. Wang, C., Kang, S.G., Lee, J., Sun, Z. \& Kim, C.H. The roles of CCR6 in migration of Th17 cells and regulation of effector T-cell balance in the gut. Mucosal Immunol. 2, 173-183 (2009).

22. Asseman, C., Read, S. \& Powrie, F. Colitogenic Th1 cells are present in the antigen-experienced T cell pool in normal mice: control by CD4+ regulatory T cells and IL-10. J. Immunol. 171, 971-978 (2003).

23. Griseri, T., Asquith, M., Thompson, C. \& Powrie, F. OX40 is required for regulatory T cell-mediated control of colitis. J. Exp. Med. 207, 699-709 (2010).

24. Haftmann, C. et al. miR-148a is upregulated by Twist1 and T-bet and promotes Th1-cell survival by regulating the proapoptotic gene Bim. Eur. J. Immunol. 45, 1192-1205 (2014).

25. Annunziato, F. et al. Phenotypic and functional features of human Th17 cells. J. Exp. Med. 204, 1849-1861 (2007).

26. Ciofani, M. et al. A validated regulatory network for Th17 cell specification. Cell 151, 289-303 (2012).

27. Duhen, R. et al. Cutting edge: the pathogenicity of IFN- $\gamma$-producing Th17 cells is independent of T-bet. J. Immunol. 190, 4478-4482 (2013).

28. Yuan, X. et al. A novel role of CD4 Th17 cells in mediating cardiac allograft rejection and vasculopathy. J. Exp. Med. 205, 3133-3144 (2008).

29. Durrant, D.M., Gaffen, S.L., Riesenfeld, E.P., Irvin, C.G. \& Metzger, D.W. Development of allergen-induced airway inflammation in the absence of T-bet regulation is dependent on IL-17. J. Immunol. 183, 5293-5300 (2009).

30. Lazarevic, V. et al. T-bet represses $T(H) 17$ differentiation by preventing Runx1-mediated activation of the gene encoding ROR $\gamma$ t. Nat. Immunol. 12, 96-104 (2011).

31. Neurath, M.F. Cytokines in inflammatory boweldisease. Nat. Rev. Immunol. 14, 329-342 (2014).

32. Lee, J.S. et al. Interleukin-23-Independent IL-17 Production Regulates Intestinal Epithelial Permeability. Immunity 43, 1-13 (2015). 
33. Maxwell, J.R. et al. Differential Roles for Interleukin-23 and Interleukin- 17 in Intestinal Immunoregulation. Immunity 43, 1-13 (2015).

34. Usui, T. et al. T-bet regulates Th1 responses through essential effects on GATA-3 function rather than on IFNG gene acetylation and transcription. J. Exp. Med. 203, 755-766 (2006).

35. Sullivan, B.M. et al. Increased Susceptibility of Mice Lacking T-bet to Infection with Mycobacterium tuberculosis Correlates with Increased IL-10 and Decreased IFN- Production. J. Immunol. 175, 4593-4602 (2005).

36. Sabet-Baktach, M. et al. Double deficiency for ROR t and T-bet drives Th2-mediated allograft rejection in mice. J. Immunol. 191, 4440-4446 (2013).

37. Intlekofer, A.M. et al. Anomalous type 17 response to viral infection by CD8 + T cells lacking T-bet and eomesodermin. Science 321, 408-411 (2008).

38. Wang, Y. et al. The transcription factors T-bet and runx are required for the ontogeny of pathogenic interferon-g-producing T helper 17 cells. Immunity 40, 1-12 (2014).

39. Bregenholt, S., Brimnes, J., Nissen, M.H. \& Claesson, M.H. In vitro activated CD4 + T cells from interferon-gamma (IFN-gamma)-deficient mice induce intestinal inflammation in immunodeficient hosts. Clin. Exp. Immunol. 118, 228-234 (1999).

40. Vonarbourg, C. et al. Regulated expression of nuclear receptor ROR $\gamma \mathrm{t}$ confers distinct functional fates to $\mathrm{NK}$ cell receptor-expressing ROR $\gamma \mathrm{t}+$ innate lymphocytes. Immunity 33, 736-751 (2010).

41. Buonocore, S. et al. Innate lymphoid cells drive interleukin-23-dependent innate intestinal pathology. Nature 464, 1371-1375 (2010).
42. Griseri, T., McKenzie, B.S., Schiering, C. \& Powrie, F. Dysregulated hematopoietic stem and progenitor cell activity promotes interleukin-23driven chronic intestinal inflammation. Immunity 37, 1-14 (2012).

43. Wang, N., Liang, H. \& Zen, K. Molecular mechanisms that influence the macrophage m1-m2 polarization balance. Front. Immunol. 5, 614 (2014).

44. Coccia, M. et al. IL-1 mediates chronic intestinal inflammation by promoting the accumulation of IL-17A secreting innate lymphoid cells and CD4 + Th17 cells. J. Exp. Med. 209, 1595-1609 (2012).

45. Corazza, N., Eichenberger, S., Eugster, H.P. \& Mueller, C. Nonlymphocytederived tumor necrosis factor is required for induction of colitis in recombination activating gene (RAG) $2\left(^{-/-}\right)$mice upon transfer of CD4 $(+)$ CD45RB(hi) T cells. J. Exp. Med. 190, 1479-1492 (1999).

46. Ahern, P.P. et al. Interleukin-23 drives intestinal inflammation through direct activity on T cells. Immunity 33, 279-288 (2010).

47. Niesner, U. et al. Autoregulation of Th1-mediated inflammation by twist1. J. Exp. Med. 205, 1889-1901 (2008).

48. Brühl, $\mathrm{H}$. et al. Targeting of $\mathrm{Gr}-1+, \mathrm{CCR} 2+$ monocytes in collageninduced arthritis. Arthritis Rheum. 56, 2975-2985 (2007).

49. Sanos, S.L. \& Diefenbach, A. Isolation of NK cells and NK-like cells from the intestinal lamina propria. Methods Mol. Biol. 612, 505-517 (2010).

50. Zimmermann, J., Radbruch, A. \& Chang, H.-D. A Ca(2+) concentration of $1.5 \mathrm{mM}$, as present in IMDM but not in RPMI, is critical for maximal response of Th cells to PMA/ionomycin. Eur. J. Immunol. 45, 1270-1273 (2015).

51. Biesen, R. et al. Sialic acid-binding Ig-like lectin 1 expression in inflammatory and resident monocytes is a potential biomarker for monitoring disease activity and success of therapy in systemic lupus erythematosus. Arthritis Rheum. 58, 1136-1145 (2008). 